\title{
The prediction of in-hospital mortality by mid-upper arm circumference: a prospective observational study of the association between mid-upper arm circumference and the outcome of acutely ill medical patients admitted to a resource-poor hospital in sub-Saharan Africa
}

\author{
Authors: Martin Otyek Opio, ${ }^{\mathrm{A}}$ Teopista Namujwiga, ${ }^{\mathrm{B}}$ Imaculate Nakitende, ${ }^{\mathrm{C}}$ John Kellett ${ }^{\mathrm{D}}$ and Mikkel Brabrand \\ on behalf of the Kitovu Hospital Study Group
}

\begin{abstract}
There are few reports of the association of nutritional status with in-hospital mortality of acutely ill medical patients in sub-Saharan Africa. This is a prospective observational study comparing the predictive value of mid-upper arm circumference (MUAC) of 899 acutely ill medical patients admitted to a resource-poor sub-Saharan hospital with mental alertness, mobility and vital signs. Mid-upper arm circumference ranged from $15 \mathrm{~cm}$ to $42 \mathrm{~cm}$, and $12(24 \%)$ of the 50 patients with a MUAC less than $20 \mathrm{~cm}$ died (OR 4.84, 95\% CI 2.23-10.37). Of the 237 patients with a MUAC more than $28 \mathrm{~cm}$ only six (2.5\%) died (OR $0.27,95 \%$ CI 0.10-0.67). On logistic regression, the National Early Warning Score (NEWS), alertness, mobility and MUAC were independent predictors of in-hospital mortality. Mid-upper arm circumference is an independent predictor of the in-hospital mortality of acutely ill medical patients in a resource-poor hospital in sub-Saharan Africa.
\end{abstract}

KEYWORDS: Predictive scores, early warning scores, nutrition, sub-Saharan Africa, in-hospital mortality

\section{Introduction}

Although there is widespread concern about the risks of obesity ${ }^{1}$ there are well-established health benefits from being well nourished and definite hazards associated with being underweight. ${ }^{2}$ Many populations in the developing world are undernourished and numerous studies have demonstrated an increased risk of death in those with low body weight. ${ }^{3,4}$ Several anthropometric

Authors: ${ }^{A}$ consultant physician and medical director, Kitovu Hospital, Masaka, Uganda; ${ }^{B}$ enrolled nurse, Kitovu Hospital, Masaka, Uganda; ' enrolled midwife, Kitovu Hospital, Masaka, Uganda; ${ }^{D}$ adjunct associate professor in acute and emergency medicine, University of Southern Denmark, Denmark; ${ }^{\mathrm{E}}$ clinical associate professor, Institute of Regional Health Research, University of Southern Denmark, Denmark measurements have been used to assess nutrition including: height, weight, body mass index (BMI), skin fold thickness etc. Mid-upper arm circumference (MUAC) is a simple, cheap and practical measure of nutrition that is recommended by the World Health Organization. It is in widespread use, particularly in children in the developing world. ${ }^{5}$ Although children ${ }^{6}$ and adults ${ }^{7,8}$ with a low MUAC are at an increased risk of dying, there are only a few reports of the association of MUAC with the in-hospital mortality of acutely ill patients.

This study reports the association between MUAC and the in-hospital mortality of 899 acutely ill medical patients treated in a resource-poor hospital in sub-Saharan Africa. It also compares MUAC with other predictors of in-hospital mortality such as age, mental alertness, functional capacity and vital signs.

\section{Methods}

This prospective observational study was performed on a 46-bed medical ward at St Joseph's Kitovu Health Care Complex, which has 220 beds and is located near Masaka, Uganda, $140 \mathrm{~km}$ from the capital city of Kampala. Together with the 330-bed Masaka Regional Referral Government Hospital, it serves Masaka Municipality (population of 79,200) and Masaka District, with a rural population of $804,300 .^{9}$

From 9 August 2016 to 16 May 2017 the clinical status and vital signs of every patient admitted to the hospital's medical unit were entered on admission and at least daily at the bedside using tablet computers into a clinical data management and decision support system (rapid electronic assessment data system [READS], Tapa Healthcare DAC) by two dedicated nurse researchers, who worked between them from 9 am to $5 \mathrm{pm}$ 7-days-a-week. All the patients admitted were acutely ill, and no patients were excluded from the study. The READS bedside assessment requires that the patient's contemporaneous mental alertness, mobility and complaints are entered each time the vital signs are measured..$^{10}$ Any patient who was not alert and calm was deemed to have altered alertness, ${ }^{11}$ and to have impaired mobility if they did not have a stable independent gait. ${ }^{12}$ The final disposition of patients was also recorded in the system. All data entries were automatically time and date stamped. In addition to the electronic READS 
assessment, the research nurses were also asked to record on paper each patient's MUAC.

The National Early Warning Score (NEWS), ${ }^{13}$ a well-validated predictor of imminent mortality, was calculated from the respiratory rate, systolic blood pressure, level of consciousness, temperature, oxygen saturations, and inspired oxygen concentration. Calculations were performed using Epi-Info version 6.0 (Centers for Disease Control and Prevention, USA), logistic regression analysis using logistic software. ${ }^{14}$ The $p$-value for statistical significance was 0.05 and was tested using Student's t-test.

Ethical approval of the study was obtained from the Ethics Committee Kitovu Hospital, which waived the need for written consent. The study is reported in accordance with the STROBE statement. ${ }^{15}$

\section{Results}

Of the 1069 patients 16 years or older admitted during the study period only 170 (15.9\%) did not have their MUAC measured.
Apart from a shorter length of hospital stay (71.4 days [SD 61.1 hours] vs 84.5 days [SD 63.0 hours], $p=0.01$ ) there were no significant differences between these patients and the final study population of 899 patients, who had a mean age of 50.3 years (SD 21.7 years) and $366(58.9 \%)$ of whom were male. On admission, 62 patients $(6.9 \%)$ had altered alertness and 384 (42.7\%) had impaired mobility. The HIV status was only known in 85 patients; 49 were positive and 36 negative. Within a mean length of stay of 84.5 days (SD 63.0 hours), 682 (75.9\%) patients were discharged independent of the care of others, $115(12.8 \%)$ were discharged dependent on others, 38 ( $4.2 \%$ ) had been transferred to another hospital, and $64(7.1 \%)$ had died.

There was no significant difference in the age, length of hospital stay or the temperature on admission of patients who died compared with those who survived until discharge. However, compared to survivors, patients who died had higher heart and respiratory rates and NEWS, and lower systolic blood pressures, oxygen saturation levels and MUAC (Table 1).

Table 1. Values of continuous variables of all patients and those who died in hospital compared with those who survived

\begin{tabular}{|c|c|c|c|c|}
\hline & Total & Alive & Dead & $\mathrm{p}$-value \\
\hline \multicolumn{5}{|l|}{ Age (years) } \\
\hline Mean (SD) & $50.3(21.7)$ & $49.9(21.5)$ & $55.2(24.1)$ & \multirow[t]{2}{*}{0.06} \\
\hline Median (Range) & $50(16-105)$ & $50.0(16-105)$ & 57.5 (19-105) & \\
\hline \multicolumn{5}{|c|}{ Length of stay (hours) } \\
\hline Mean (SD) & $84.5(63.0)$ & $84.3(61.6)$ & $87.0(79.3)$ & \multirow[t]{2}{*}{0.74} \\
\hline Median (Range) & $73(1-551)$ & $73(1-551)$ & $70(3-356)$ & \\
\hline \multicolumn{5}{|l|}{ Heart rate (bpm) } \\
\hline Mean (SD) & 86.5 (18.9) & $85.6(17.8)$ & $97.8(27.4)$ & \multirow[t]{2}{*}{$<0.00001$} \\
\hline Median (Range) & $84(38-190)$ & $84(38-190)$ & $98(48-158)$ & \\
\hline \multicolumn{5}{|c|}{ Respiratory rate (bpm) } \\
\hline Mean (SD) & $23.1(6.8)$ & $22.5(6.1)$ & $30.6(10.1)$ & \multirow[t]{2}{*}{$<0.00001$} \\
\hline Median (Range) & $22(12.0-50.0)$ & $21.0(12.0-50.0)$ & $27.0(15.0-50.0)$ & \\
\hline \multicolumn{5}{|l|}{ SBP $(\mathrm{mmHg})$} \\
\hline Mean (SD) & $112.7(0.9)$ & $113.3(24.9)$ & $105.3(31.6)$ & \multirow[t]{2}{*}{0.02} \\
\hline Median (Range) & $110.0(50.0-214.0)$ & $111.0(50.0-214.0)$ & $100.0(50.0-190.0)$ & \\
\hline \multicolumn{5}{|l|}{ Temperature $\left({ }^{\circ} \mathrm{C}\right)$} \\
\hline Mean (SD) & $36.8(1.0)$ & $36.8(0.9)$ & $36.9(2.2)$ & \multirow[t]{2}{*}{0.20} \\
\hline Median (Range) & $36.7(24.0-50.0)$ & $36.7(24.0-41.1)$ & $36.5(29.0-50.0)$ & \\
\hline \multicolumn{5}{|c|}{ Oxygen saturation (\%) } \\
\hline Mean (SD) & $94.5(7.3)$ & $95.0(6.4)$ & $88.3(13.6)$ & \multirow[t]{2}{*}{$<0.00001$} \\
\hline Median (Range) & 97 (36-99) & 97 (38-99) & 92 (36-99) & \\
\hline \multicolumn{5}{|l|}{ NEWS } \\
\hline Mean (SD) & $4.1(3.1)$ & $3.8(2.8)$ & $8.4(3.2)$ & \multirow[t]{2}{*}{$<0.00001$} \\
\hline Median (Range) & $4.0(0-17)$ & $3.0(0-14)$ & $8.0(0-17)$ & \\
\hline \multicolumn{5}{|l|}{ MUAC (cm) } \\
\hline Mean (SD) & $26.1(4.3)$ & $26.3(4.3)$ & $23.9(3.6)$ & \multirow[t]{2}{*}{0.00002} \\
\hline Median (Range) & $26.0(15.0-43.0)$ & $26.0(15.0-43.0)$ & $23.5(16.0-31.5)$ & \\
\hline
\end{tabular}

bpm = beats or breaths per minute; $\mathrm{MUAC}=$ mid-upper arm circumference; NEWS = National Early Warning Score; SBP = systolic blood pressure; $\mathrm{SD}=$ standard deviation 


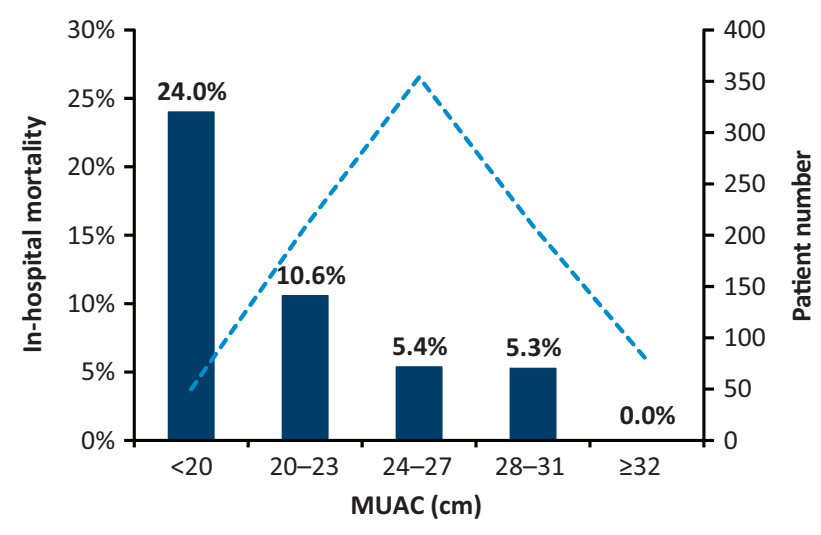

In-hospital mortality _- - Patient number

Fig 1. In-hospital mortality according to mid-upper arm circumference (MUAC) in centimetres $(\mathrm{cm})$.

Mid-upper arm circumference ranged from $15 \mathrm{~cm}$ to $42 \mathrm{~cm}$. Twelve (24\%) of the 50 patients with a MUAC less than $20 \mathrm{~cm}$ died; however, none of the 78 patients with a MUAC $\geq 32 \mathrm{~cm}$ died (Fig 1). A MUAC below $20 \mathrm{~cm}$ and a NEWS $\geq 7$ had the highest chi-square value for in-hospital mortality (OR 4.8, 95\% CI 2.23-10.37). In contrast, nearly a quarter of the patients had a MUAC $>28 \mathrm{~cm}$ and were significantly less likely to die (OR 0.27, 95\% CI 0.10-0.67). Although men had a significantly lower MUAC than women ( $25.5 \mathrm{~cm}$ [SD 3.7] vs $26.6 \mathrm{~cm}$ [SD 4.7], $\mathrm{p}=0.0001$ ), the values associated with mortality that had the highest chi-square values were the same for both sexes. The other categorical variables associated with increased mortality were the use of supplemental oxygen, altered alertness and impaired mobility, but not gender (Table 2).

Logistic regression revealed that altered alertness, impaired mobility, NEWS and MUAC (either as continuous or categorical variables) were independent predictors of in-hospital mortality, whereas age, gender, the use of supplement oxygen, oxygen saturation levels and the individual vital signs were not (Table 3).

\section{Discussion}

This study shows that MUAC is a predictor of the in-hospital mortality of acutely ill medical patients in sub-Saharan Africa, which is independent of age, gender, mental alertness, impaired mobility and vital signs. Patients with a MUAC $<20 \mathrm{~cm}$ were almost
Table 3. Odds ratio for in-hospital mortality of the four parameters found by logistic regression to be independent predictors, calculated with NEWS and MUAC as either categorical or continuous variables

$\begin{array}{lcl}\text { Parameter } & \text { Odds ratio }(\mathbf{9 5 \%} \mathrm{CI}) & \text { p-value } \\ \text { NEWS and MUAC as categorical parameters } & \\ \text { NEWS } \geq \mathbf{7} & 10.75(5.63-20.56) & <0.00001 \\ \text { MUAC }<20 \mathrm{~cm} & 3.45(1.46-8.16) & 0.0049 \\ \text { Altered alertness } & 3.70(1.79-7.64) & 0.0004 \\ \text { Impaired mobility } & 3.72(1.76-7.89) & 0.0006\end{array}$

Hosmer-Lemeshow goodness-of-fit p-value of 0.19

NEWS and MUAC as continuous parameters

$\begin{array}{lll}\text { NEWS } & 1.44(1.30-1.60) & <0.00001 \\ \text { MUAC (cm) } & 0.92(0.85-0.99) & 0.03 \\ \text { Altered alertness } & 2.75(1.33-5.71) & 0.007 \\ \text { Impaired mobility } & 3.32(1.56-7.05) & 0.002 \\ \text { Hosmer-Lemeshow goodness-of-fit p-value of } 0.20 & \end{array}$

See text for definitions of 'Altered alertness' and 'Impaired mobility'. $\mathrm{MUAC}=$ mid-upper arm circumference; NEWS = National Early Warning Score

five times more likely to die in hospital, and those with a MUAC $>28 \mathrm{~cm}$ were almost four times more likely to survive.

This is a small single-centre study, performed in a resourcepoor hospital in sub-Saharan Africa that only reports in-hospital mortality; patient diagnoses and long term outcomes were not examined. Since diagnostic investigations were limited, and no autopsies were performed, any diagnoses made would have been based on local expert opinion. In contrast, the parameters examined in this study are all objective and require little skill to measure. HIV status was known in only a small number of patients. However, there was no difference in the MUAC or in-hospital mortality of those patients known to be HIV positive compared with those known to be HIV negative. As far as we know, there was no selection bias for the patients who did not have their MUAC measured. Both researchers worked from 9 am to $5 \mathrm{pm}$ each day, so the time of admission would not have been an issue. However, patients who did not have their MUAC recorded did have a shorter length of stay, so if the measurement was overlooked at the time of the first assessment, the patient

Table 2. In-hospital mortality associated with categorical variables

\begin{tabular}{llllll} 
Parameter & $\mathbf{n}$ & In-hospital mortality & Odds ratio (95\% CI) & Chi-square & p-value \\
NEWS $\geq \mathbf{7}$ & $193(21.5 \%)$ & $25.9 \%$ & $17.28(8.93-33.95)$ & 127.6 & $<0.00001$ \\
Altered alertness & $62(6.9 \%)$ & $33.9 \%$ & $9.46(4.89-18.24)$ & 67.8 & $<0.00001$ \\
Impaired mobility & $384(42.7 \%)$ & $14.1 \%$ & $8.26(3.97-17.66)$ & 47.1 & $<0.00001$ \\
Supplemental oxygen & $52(5.8 \%)$ & $25.0 \%$ & $5.20(2.45-10.92)$ & 23.9 & $<0.00001$ \\
MUAC < $\mathbf{2 0} \mathbf{~ c m}$ & $50(5.6 \%)$ & $24.0 \%$ & $4.84(2.23-10.37)$ & 20.2 & $<0.00001$ \\
MUAC > $\mathbf{2 8} \mathbf{~ c m}$ & $237(26.4 \%)$ & $2.5 \%$ & $0.27(0.10-0.67)$ & 9.3 & 0.002 \\
Male & $366(40.7 \%)$ & $8.2 \%$ & $1.31(0.76-2.26)$ & 0.8 & 0.36 \\
\hline See text for definitions of 'Altered alertness' and 'Impaired mobility'. & &
\end{tabular}


may have been discharged before there was time to correct the oversight.

This study examined the association between in-hospital mortality and MUAC in our patient population. There are, of course, many factors that influence MUAC, including malnutrition and poverty; all of our patients were poor. Diagnoses are also major confounding factors. Although patient diagnoses were not studied, common conditions and causes of mortality in our patient population are HIV-related illness, diabetes and tuberculosis; ${ }^{9}$ all these conditions are associated with weight loss. Therefore, it is possible that the relationship between low MUAC and mortality may be explained by reverse causation (ie mortality was not caused by the low MUAC, but by the underlying illnesses that caused both weight loss and the patient to die). ${ }^{4}$ Similarly, patients with a high MUAC would have less chance of suffering from fatal diseases that cause weight loss.

It might be assumed that MUAC measurement in acutely ill patients will be of less value in the developed world, where tuberculosis and HIV are relatively uncommon. However, PowellTuck and Hennessy reported that a low MUAC predicted mortality in acutely ill patients attending the Royal London Hospital better than their body mass index. ${ }^{16}$ They did not find, however, that an increased MUAC was associated with a reduced mortality. Indeed, a long term follow-up study has reported that both a high and low MUAC are associated with an increased mortality. Nevertheless, there is increasing evidence that moderate obesity improves the survival of sick patients. This reverse epidemiology was first reported in renal dialysis patients, ${ }^{17}$ but has since been observed in heart failure, ${ }^{18}$ the elderly, ${ }^{19}$ chronic obstructive pulmonary disease (COPD) ${ }^{20}$ and intensive care patients with sepsis, pneumonia ${ }^{21}$ and acute lung injury. ${ }^{22}$ It is possible, therefore, that what might be considered as excessive nutrition provides a survival benefit to patients with infection and other acute illness.

Regardless of whether or not a high MUAC is protective and a Iow MUAC causes mortality, the measurement is easy to perform and a powerful independent predictor of in-hospital outcome.

It is a quick, simple and cheap observation that can be carried out anywhere by a single operator, and requires only a tape measure and little training or expertise to perform. ${ }^{8}$ The measurement of MUAC is widespread in African paediatric wards, and both of our nurses were familiar with the technique. Moreno et al have studied its interobserver and intraobserver error in adolescents and found an interobserver technical error of measurement of $0.47 \mathrm{~cm}$ and an interobserver coefficient of reliability of $97 \%{ }^{23}$ In contrast, accurately measuring height and body weight to calculate body mass index can be extremely difficult in bedridden, severely ill patients. Moreover, there is some evidence that since MUAC measures both fat and muscle, it may be a better indicator of nutritional status. ${ }^{8}$

\section{Conclusion}

Mid-upper arm circumference is confirmed as a powerful independent predictor of the in-hospital mortality of acutely ill medical patients in a resource-poor hospital in sub-Saharan Africa. It can be measured quickly and easily at no cost. A review of the available literature suggests that it should be valuable in the risk assessment of acute illness in other patient populations.

\section{Acknowledgements}

The authors wish to acknowledge and thank Tapa Healthcare DAC (Dundalk, Ireland) for the complimentary use of their Rapid Electronic Assessment Data system.

\section{Funding and conflicts of interest}

All other costs were borne by the authors. John Kellett is a major shareholder, director and chief medical officer of Tapa Healthcare DAC.

\section{Consent}

This was an observational study that is part of an ongoing quality improvement project. This study conformed to the principles outlined in the Declaration of Helsinki and was approved by the hospital's ethics committee: assessment of nutritional status on all patients is strongly encouraged by the Ugandan Ministry of Health and no patients refused measurement of their mid-upper arm circumference which is part of routine practice.

\section{References}

1 The Global Burden of Disease 2015 Obesity Collaborators. Health effects of overweight and obesity in 195 countries over 25 years. N Engl J Med 2017;377:13-27.

2 Flegal KM, Graubard BI, Williamson DF, Gail MH. Excess deaths associated with underweight, overweight, and obesity. JAMA 2005:293:1861-7.

3 Klenk J, Nagel G, Ulmer H et al. Body mass index and mortality: results of a cohort of 184,697 adults in Austria. Eur ] Epidemiol 2009;24:83-91.

4 Roh L, Braun J, Chiolero A et al. Mortality risk associated with underweight: a census-linked cohort of 31,578 individuals with up to 32 years of follow up. BMC Public Health 2014;14:371.

5 WHO. Child growth standards. Arm circumference for age. www.who.int/ childgrowth/standards/ac_for_age/en [Accessed 21 February 2018].

6 Rasmussen J, Andersen A, Fisker AB et al. Mid-upper-armcircumference and mid-upper-arm circumference $z$-score: the best predictor of mortality? Eur J Clin Nutr 2012;66:998-1003.

7 de Hollander EL, Bemelmans WJ, de Groot LC. Associations between changes in anthropometric measures and mortality in old age: a role for mid-upper arm circumference? J Am Med Dir Assoc 2013;14:187-93.

8 Wijnhoven HA, van Bokhorst-de van der Schueren MA, Heymans $\mathrm{MW}$ et al. Low mid-upper arm circumference, calf circumference, and body mass index and mortality in older persons. J Gerontol $A$ Biol Sci Med Sci 2010;65:1107-14.

9 Nabayigga B, Kellett J, Opio MO. The alertness, gait and mortality of severely ill patients at two months after admission to a resource poor sub-Saharan hospital - Why is post-discharge surveillance not routine everywhere? Eur J Intern Med 2016;28:25-31.

10 Hickey A, Gleeson M, Kellett J. READS: the rapid electronic assessment documentation system. Br J Nurs 2012;21:1333-40.

11 McNarry AF, Goldhill DR. Simple bedside assessment of level of consciousness: comparison of two simple assessment scales with the Glasgow Coma scale. Anaesthesia 2004;59:34-7.

12 Kellett J, Clifford M, Ridley A, Murray A, Gleeson M. A four item scale based on gait for the immediate global assessment of acutely ill medical patients - one look is more than 1000 words. Eur Geriatr Med 2014;5:92-6.

13 Royal College of Physicians. National Early Warning Score (NEWS):standardisingthe assessment of acute illness severity in the NHS. Report of a Working Party. London: RCP, 2012.

14 Dallal GE. LOGISTIC: a logistic regression program for the IBM PC. Am Stat 1988;42:272. 
15 Vandenbroucke JP, von Elm E, Altman DG et al. Strengthening the Reporting of Observational Studies in Epidemiology (STROBE): explanation and elaboration. Epidemiology 2007;18:805-35.

16 Powell-Tuck J, Hennessy EM. A comparison of mid upper arm circumference, body mass index and weight loss as indices of undernutrition in acutely hospitalised patients. Clin Nutr 2003;22:307-12.

17 Kalantar-Zadeh K, Block G, Humphreys MH, Kopple JD. Reverse epidemiology of cardiovascular risk factors in maintenance dialysis patients. Kidney Int 2003;63:793-808.

18 Kalantar-Zadeh K, Block G, Horwich T, Fonarow GC. Reverse epidemiology of conventional cardiovascular risk factors in patients with chronic heart failure. J Am Coll Cardiol 2004;43:1439-44.

19 Veronese N, Cereda E, Solmi M et al. Inverse relationship between body mass index and mortality in older nursing home residents: a meta-analysis of 19,538 elderly subjects. Obes Rev 2015;16:1001-15.

20 Cao C, Wang R, Wang J et al. Body mass index and mortality in chronic obstructive pulmonary disease: a meta-analysis. PLoS One 2012;7:e43892.
21 Pepper DJ, Sun J, Welsh ] et al. Increased body mass index and adjusted mortality in ICU patients with sepsis or septic shock: a systematic review and meta-analysis. Crit Care 2016;20:181.

$22 \mathrm{Ni}$ YN, Luo J, Yu H et al. Can body mass index predict clinical outcomes for patients with acute lung injury/acute respiratory distress syndrome? A meta-analysis. Crit Care 2017;21:36.

23 Moreno LA, Joyanes M, Mesana MI et al. Harmonisation of anthropometric measurements for a multicenter nutrition survey in Spanish adolescents. Nutrition 2003;19:481-6.

Address for correspondence: Dr John Kellett, Department of Emergency Medicine, Hospital of South West Jutland, Finsensgade 35, 6700 Esbjerg, Denmark.

Email:jgkellett@eircom.net

\section{Assessing trainees in the workplace An e-learning module for secondary care doctors}

Three hours of CPD-approved interactive learning covering:

$>$ feedback

> supervised learning events (SLES)

$>$ workplace-based assessments (WPBAs)

$>$ the role of the Annual Review of Competence Progression (ARCP).

For more information please visit: www.rcplondon.ac.uk/elearning

\section{Royal College} of Physicians

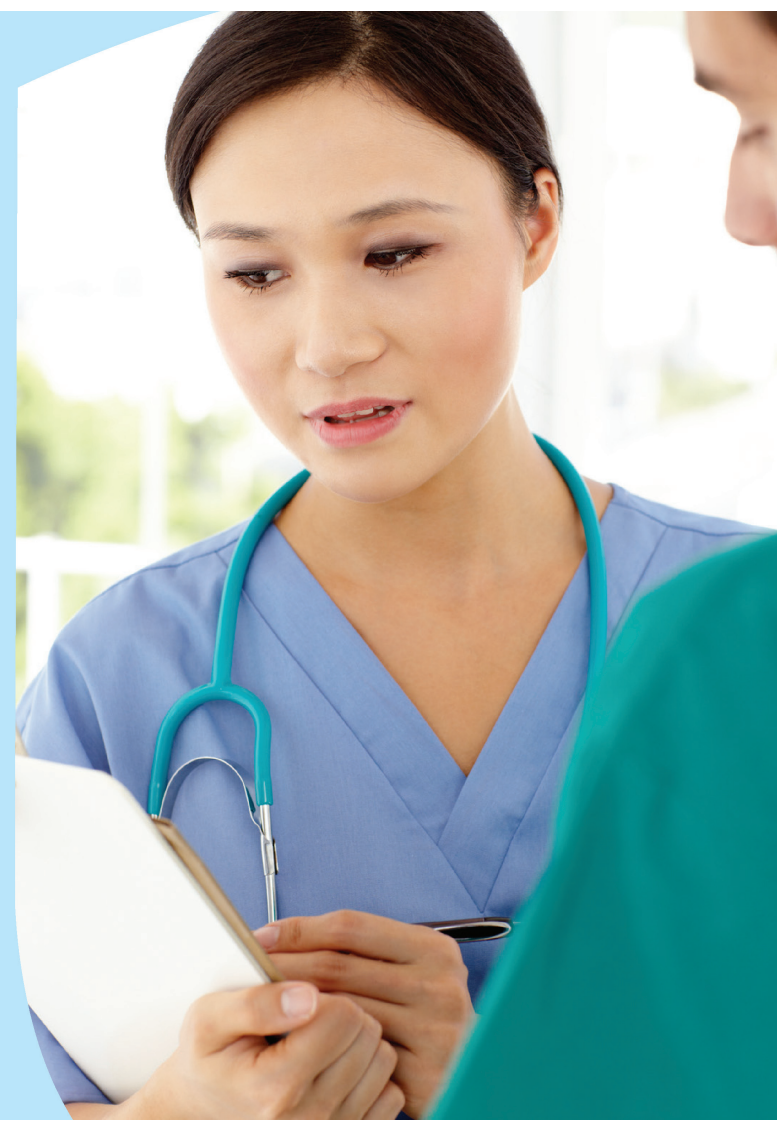

